Sánchez-Pay, A.; García-Castejón, A.; Courel-lbáñez, J.; Sánchez-Alcaraz, B.J. (2020). Influence of Low-Compression Balls in Padel Initation Stage. Revista Internacional de Medicina y Ciencias de la Actividad Física y el Deporte vol. 20 (79) pp. 419-434 Http://cdeporte.rediris.es/revista/revista79/artinfluencia1174.htm

DOI: http://doi.org/10.15366/rimcafd2020.79.003

\title{
ORIGINAL
}

\section{INFLUENCIA DE LAS PELOTAS DE BAJA PRESIÓN EN LA INICIACIÓN AL PÁDEL}

\section{INFLUENCE OF LOW-COMPRESSION BALLS IN PADEL INITATION STAGE}

\author{
Sánchez-Pay, A. ${ }^{1}$; García-Castejón, A. ${ }^{2}$; Courel-Ibáñez, J.. ${ }^{1}$ y Sánchez- \\ Alcaraz, B.J. ${ }^{1}$ \\ 1 Doctor en Ciencias de la Actividad Física y el Deporte. Facultad de Ciencias del Deporte. \\ Universidad de Murcia (España) aspay@um.es, courel@um.es, bjavier.sanchez@um.es \\ ${ }^{2}$ Graduado en Ciencias de la Actividad Física y el Deporte. Facultad de Ciencias del Deporte. \\ Universidad de Murcia (España) albeertogarciaa@gmail.com
}

Código UNESCO / UNESCO code: 5899. Otras Especialidades Pedagógicas (Educación Física y Deporte)

Clasificación Consejo de Europa / Concil of Eurpe classification: 4. Educación Física Y Deporte Comparado / Physical Education And Sport Compared

Recibido 31 de agosto de 2018 Received August 31, 2018

Aceptado 31 de diciembre de 2019 Accepted December 31, 2019

\section{RESUMEN}

El objetivo de este estudio fue analizar las demandas físicas de la competición en pádel de iniciación, y observar la influencia de diferentes tipos de pelota Dieciséis jugadores de pádel en iniciación participaron en este estudio. Cada judador portó un pulsómetro durante el partido. Se utilizaron cuestionarios al término de cada partido para evaluar el esfuerzo percibido y la satisfacción. Los resultados no mostraron diferencias significativas en la estructura temporal de los partidos. Se encontraron diferencias $(p<0,001)$ entre los partidos con pelota normal y pelota de baja presión tanto en la frecuencia cardiaca media $(145,43$ ppm vs 140,19 ppm) como en el \%FCmáx (72,47 vs 69,85$)$. Los jugadores mostraron mayor satisfacción en el uso de pelotas de baja presión. Estos datos pueden ser de utilidad para determinar el uso de un tipo de pelota u otra y mejorar la planificación del entrenamiento específico en jóvenes jugadores de pádel 
PALABRAS CLAVE: Deportes de raqueta, equipamiento, formación, competición.

\section{ABSTRACT}

The aim of this study was to analyze the physical demands in padel initiation stage and to observe the influence of playing with different type of padel balls. Sixteen padel players at initiation stage participated in this study. Players wore a heart rate (HR) monitor. Perceived exertion and satisfaction questionnaires were administrated at the end of the matches. No differences were found in the match temporal structure. Playing with official balls caused a significant increase in HR $(p<0.001)$ both in average and maximal values (Average HR: $145 \mathrm{bpm}$, \%HRmax: 72.5\%) comparing to low-compression balls (Average HR: $140 \mathrm{bpm}$ \%HRmax: 69.9\%). In addition, players showed higher satisfaction when using low-compression balls. This information may have implications to determine the use of normal balls or low-compression balls and the design of specific training programmes for young padel players

KEY WORDS: Racquet sports, equipment, education, competition.

\section{INTRODUCCIÓN}

El pádel es una nueva modalidad deportiva dentro de los deportes de raqueta (Sánchez-Alcaraz, 2013). En los últimos años, este deporte ha experimentado un crecimiento exponencial debido a su carácter lúdico, fácil de practicar y abierto a cualquier edad o sexo (Courel-Ibañez, Sánchez-Alcaraz, García, y Echegaray, 2017). Además, cada vez se construyen más instalaciones de pádel en nuestro país (Muñoz-Marín et al., 2016), y aumentan el número de investigaciones relacionadas con este deporte (Sánchez-Alcaraz, Cañas y Courel-Ibáñez, 2015). La inclusión de deportes de raqueta modernos como el pádel dentro del ámbito escolar se presenta como una alternativa a los deportes tradicionales y una nueva actividad física para que los alumnos practiquen durante su tiempo de ocio (Díaz, Cantó, y Soto, 2013).

En pádel, son numerosas las investigaciones que han analizado su estructura temporal, como el tiempo de juego, el tiempo de descanso y el tiempo total de un partido. En jóvenes jugadores, la duración media de un punto de pádel se sitúa alrededor de 7 segundos, con un tiempo de descanso entre puntos de 9 segundos (Courel-lbáñez, Sánchez-Alcaraz y Cañas, 2017; Sañudo, De Hoyo y Carrasco, 2008), aunque estos datos pueden variar en función del género, la edad, el nivel de práctica, la diferencia en el marcador o la importancia del punto (Courel-Ibáñez y Sánchez-Alcaraz , 2017; Pradas, Castellar, Coll, y Rapún, 2012; Sánchez-Alcaraz, Courel-Ibáñez, Díaz, Grijota y Muñoz, 2019). En competiciones de pádel profesional la duración de los puntos es mayor (entre 10 y 14 segundos), tanto en hombres como en mujeres (Almonacid, 2012; Althaus, 
2012, Torres-Luque, Ramirez, Cabello-Manrique, Nikolaidis y Alvero-Cruz, 2015).

Basándonos en investigaciones previas relacionadas con las demandas de la competición en pádel y deportes de raqueta, podríamos definir el pádel como un deporte con unas exigencias físicas de carácter intermitente, en el que se encadenan esfuerzos de alta intensidad y corta duración con descansos a una baja intensidad (Sánchez-Alcaraz, Courel-lbáñez y Cañas, 2018). Atendiendo a las demandas fisiológicas del pádel, varios estudios han mostrado que la frecuencia cardiaca media durante el juego es de $148 \pm 13,7$ ppm, lo que supone un porcentaje del $73,9 \pm 4,65$ respecto a la $\mathrm{FC}$ máxima registrada en el laboratorio (Sañudo et al., 2008).

El pádel es un deporte abierto en la que será fundamental una toma de decisiones constante, debido a que los jugadores deben resolver diferentes situaciones que se producen durante el juego (Courel-lbáñez y Sánchez-Alcaraz, 2018). En la iniciación deportiva, diferentes estudios han indicado la necesidad de adaptar la competición a las características de los jugadores, modificando las reglas del juego, el equipamiento deportivo y el espacio de juego, desde un enfoque constructivista. En tenis, estas modificaciones han mejorado el rendimiento y el aprendizaje de los jóvenes jugadores (Sánchez-Alcaraz, 2013c), incrementando su participación y disfrute (Buszard, Reid, Masters, y Farrow, 2016). Un tamaño de la pista menor y el uso de pelotas de baja presión, también parece favorece la precisión y la potencia de determinados golpes (Larson y Guggenheimer, 2013). En pádel, la modificación del material deportivo (pelotas de menor presión y palas más reducidas) y espacio de juego (pistas reducidas) mejoró algunos aspectos técnico-tácticos como el juego de fondo y red y los golpes de definición (Lacasa, Orteub, Gabriel, Torrents, y Salas, 2017).

Algunas investigaciones han mostrado también como bajar la altura de la red puede mejorar el juego ofensivo y aumentar el número de los golpes ganadores (Sánchez-Pay, Sánchez-Alcaraz, Courel-lbáñez, 2018). Sin embargo, disminuir la altura de la red reduce el tiempo de juego en cada punto, ya que se dificulta el golpeo posterior del oponente (Limpens, Buszard, Shoemaker, Saverlsbergh, y Reid, 2018). Por lo tanto, es muy importante adaptar la altura de la red a la edad o el nivel de los jugadores, debido a que una altura más baja puede incrementar el número de errores no forzados por un impacto de pelota más bajo del jugador, además de reducir el juego en el fondo de la pista (Timmerman et al., 2014).

En pádel, la modificación del tamaño de la pista o la altura de la red es difícil, ya que este deporte se juega en unas pistas cerradas en los laterales y fondos por paredes de cristal y malla electrosoldada. Por lo tanto, la adaptación más sencilla en el proceso de enseñanza-aprendizaje en la iniciación al pádel es el uso de pelotas de baja presión. La utilización de pelotas con un $75 \%$ de la presión oficial en tenis ha incrementado la duración de los puntos, el número de golpes ganadores y totales, el porcentaje de acierto en el saque y reducido el número de errores en comparación con las pelotas normales (Kachel, Buszard, y Reid, 2014). Además, los jugadores se sintieron más cómodos y satisfechos cuando 
se adaptó el material deportivo a sus características físicas (Farrow y Reid, 2010). De este modo, parece que el uso de pelotas vedes de baja presión en pádel podría mejorar el rendimiento en jóvenes jugadores.

Sin embargo, no se han encontrado estudios que analicen la influencia de las pelotas de baja presión en las acciones técnico-tácticas en la competición y las demandas fisiológicas en jóvenes jugadores de pádel. Por lo tanto, el objetivo de este estudio fue analizar las demandas físicas y fisiológicas en la iniciación al pádel en función del uso de pelotas normales y de pelotas de baja presión.

\section{MATERIAL Y MÉTODOS}

\subsection{Diseño}

Se ha utilizado una metodología de tipo cuantitativo que responde a un estudio cuasi-experimental de carácter transversal donde se combinan los análisis de tipo descriptivo y los de relación.

\subsection{Participantes}

La muestra estuvo compuesta por 16 jugadores de pádel de nivel de iniciación (10 niños y 6 niñas) de características similares (edad $=10 \pm 0,8$ años; altura $=$ $146,0 \pm 4,9 \mathrm{~cm}$; peso $=37,4 \pm 7,3 \mathrm{~kg}$ ). Los jugadores entrenaban 2 horas semanales y tenían una experiencia en la competición deportiva de un año. El estudio contó con la aprobación del Comité de Ética de la Universidad de Murcia.

\subsection{Instrumentos}

Para la filmación de los partidos se utilizaron 4 videocámaras gran angular, colocadas sobre un trípode cada una, situadas en trípodes a 2,5 metros de la pista, detrás de la pared de fondo. La disposición de la cámara permitía la visión total de las zonas del campo. Para el análisis del video se utilizó el software Lince (Gabin, Camerino, Anguera, y Castañer, 2012). Este software de uso libre permite la creación de una hoja de registro para analizar diferentes acciones del juego, tanto temporales como gestuales.

Para poder medir el ritmo cardiaco, todos los jugadores fueron equipados con una cinta conectada por bluetooth a un monitor de telemetría Polar Team $2^{\circledR}$ (Polar, Kempele, Finlandia) donde se registró la frecuencia cardiaca (FC) desde antes del calentamiento. La FC se registró cada segundo desde el inicio hasta el final del partido. Los datos se analizaron con el software Polar Team $2^{\circledR}$, indicando los valores medios, mínimos y máximos de la FC, excluyendo valores atípicos (resultados de FC de 0 o >220). Se estimó el porcentaje sobre la FC máxima (\%FCmáx) para cada participante a través de la fórmula $208-(0,7 x$ edad) propuesta por Tanaka, Monahan, y Seals (2001), por ser la fórmula que mejor se adapta a este tipo de población (Machado y Denadai, 2011). Dicha estimación permitió la determinación de la intensidad para cada participante 
durante cada condición de actividad utilizando los rangos de intensidad marcados por el ACSM (American College of Sports Medicine, 2014).

Se evaluó la percepción subjetiva del esfuerzo (RPE) a través de la escala 6-20 de Borg (Borg y Kaijser, 2006). Además, se les administró un cuestionario de satisfacción que incluía tres preguntas: 1) ¿Con qué tipo de pelota te has divertido más?; 2) ¿Con qué tipo de pelota crees que los puntos han sido más largos?; 3) ¿Con qué tipo de pelota te ha resultado más fácil y cómodo jugar?; todas ellas con una única respuesta "a) Primera pelota" o "b) Segunda pelota".

\subsection{Procedimiento}

Siguiendo con los principios de la Declaración de Helsinki, los jugadores participaron voluntariamente en el estudio, y se obtuvo un consentimiento informado por parte de los padres o tutores de todos los participantes. Se jugaron y grabaron un total de ocho sets de pádel, con un descanso de 15 minutos entre sets. Los cuatro primeros sets se jugaron con una pelota oficial (Head Pádel ${ }^{\circledR}$ ) y los siguientes con una pelota de baja presión de punto verde (Head punto verde $^{\circledR}$ ). La pelota de punto verde tenía un $25 \%$ menos de presión que la pelota oficial. Para la organización de los partidos se organizaron ocho parejas de un nivel similar en cuatro partidos diferentes. Antes de comenzar los partidos, los jugadores realizaron un calentamiento general de 5 minutos que consistió en movilidad articular y carrera continua y un calentamiento específico que incluía golpes de fondo, golpes de red y saques, con una duración de 5 minutos. Todos los partidos fueron grabados, y cada participante portó un pulsómetro para registrar la FC durante todo el partido. Tras finalizar cada set, los jugadores rellenaron los cuestionarios de esfuerzo percibido y de satisfacción de forma anónima.

\subsection{Análisis de datos}

Para el visionado del partido y la codificación del mismo se siguió el proceso de observación sistemática de dos observadores. En primer lugar, se realizó el entrenamiento de los observadores. Posteriormente los observadores codificaron un set y se compararon con un observador experimentado para calcular la fiabilidad intra-observador. Tras una semana, cada observador volvió a registrar el mismo set para calcular la fiabilidad inter-observador. Se utilizó el Kappa de Cohen's, obteniendo valores por encima de >0,87 en todas las variables, considerándose como resultados muy buenos (Landis y Koch, 1977).

Se diseñó una hoja de cálculo (Microsoft Excel) donde se recogieron los datos y posteriormente fueron exportados al paquete informático SPSS v.21.0 para Windows (IBM Corp., Armonk, NY, USA). Se realizó un análisis descriptivo para calcular la media (M) mínimos (Min), máximos (Máx) y desviación típica (DT) de cada una de las variables. Posteriormente se confirmó la normalidad de cada variable y se utilizó la prueba $t$ de Student para determinar las posibles diferencias de cada variable según el tipo de pelota (oficial y baja presión). 
Además, se utilizó la función recuento para conocer el tiempo de participación de cada jugador sobre las diferentes zonas de intensidad. Se utilizó la prueba T Student para conocer las posibles diferencias entre el tiempo de participación en cada zona de intensidad en función del tipo de pelota. Para el análisis de los cuestionarios se realizó un recuento de las respuestas. La significación estadística se estableció en $\mathrm{p}<0,05$.

\section{RESULTADOS}

En la tabla 1 se presentan los estadísticos descriptivos de la duración del set, número de puntos por set y número de golpes por set con pelotas oficiales y con pelotas de baja presión.

Tabla 1. Valores mínimos (Mín), máximos (Máx), medios (M), desviación típica (DT), y significación ( $p)$, de los sets analizados.

\begin{tabular}{lcccccccccc}
\hline & \multicolumn{9}{c}{ Pelota oficial } & \multicolumn{6}{c}{ Pelota de baja presión } & \multirow{2}{*}{ Dif. } \\
\cline { 2 - 9 } & Mín & Máx & M & DT & Mín & Máx & M & DT & $p$ \\
\cline { 2 - 9 } Duración Set & 15,08 & 33,57 & 24,03 & 8,38 & 17,51 & 28,38 & 23,38 & 5,06 & 0,51 & 0,922 \\
№ Puntos set & 31,00 & 76,00 & 51,75 & 20,21 & 35,00 & 65,00 & 48,25 & 12,55 & 3,25 & 0,791 \\
№ Golpes set & 122,00 & 343,00 & 223,25 & 91,03 & 168,00 & 284,00 & 209,00 & 52,19 & 9,75 & 0,841 \\
\hline
\end{tabular}

Leyenda: №: Número. Dif: Diferencia de Medias.

Aunque no se encontraron diferencias estadísticamente significativas en la duración del set y el número de puntos y golpes por set, los sets jugados con pelotas normales tuvieron una duración más elevada y un mayor numero de golpes y puntos.

En la tabla 2, se muestran los estadísticos descriptivos del número de golpes por punto, diferenciando los golpes con bote y los golpes sin bote, además de la duración de cada punto. No se encontraron diferencias estadísticamente significativas entre los valores analizados. Los valores medios, mínimos y máximos se muestran muy similares para los dos tipos de pelota utilizada.

Tabla 2. Valores mínimos (Mín), máximos (Máx), medios (M), desviación típica (DT), y significación (p) por punto.

\begin{tabular}{cccccccccccc}
\hline & \multicolumn{9}{c}{ Pelota oficial } & \multicolumn{7}{c}{ Pelota de baja presión } & \multirow{2}{*}{ Dif. } & \\
\cline { 2 - 9 } & Mín & Máx & M & DT & Mín & Máx & M & DT & & $\mathrm{p}$ \\
\cline { 2 - 9 } № Golpes punto & 1,00 & 19,00 & 4,31 & 2,76 & 1,00 & 18,00 & 4,33 & 2,61 & $-0,01$ & 0,948 \\
Gs. con bote & 1,00 & 13,00 & 3,81 & 2,13 & 1,00 & 15,00 & 3,86 & 2,18 & $-0,04$ & 0,819 \\
Gs. sin bote & 1,00 & 7,00 & 1,64 & 1,20 & 1,00 & 4,00 & 1,49 & 0,83 & 0,14 & 0,434 \\
D. del punto & 1,13 & 32,49 & 8,07 & 4,79 & 0,93 & 31,28 & 7,99 & 4,63 & 0,08 & 0,859 \\
\hline
\end{tabular}

Leyenda: №: Número; Gs: Golpes; D: Duración. Dif: Diferencia de Medias. 
En la figura 1 se muestran los porcentajes de la distribución del número de golpes presentes en cada punto de juego. El $70 \%$ de los puntos se finalizaron realizando entre 2 y 5 golpeos. Únicamente el $30 \%$ de los puntos tuvieron más de 5 golpeos.

\section{Distribución del número de golpes por punto}

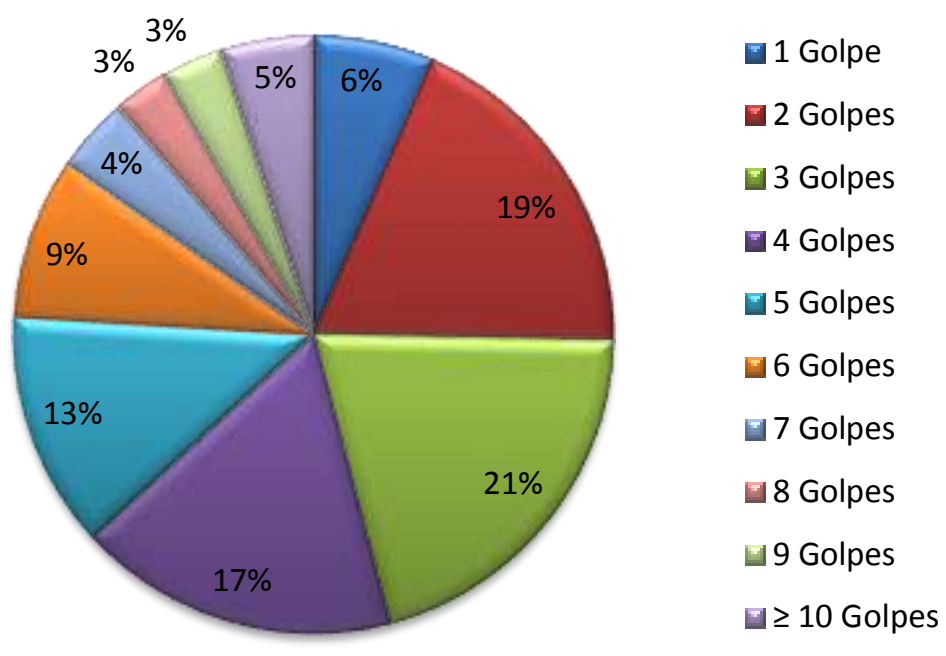

Figura 1. Distribución del \% número de golpes por punto.

En la figura 2 se presenta el porcentaje del tiempo de juego y de descanso, obteniendo menos del $30 \%$ del tiempo de trabajo durante el partido de pádel.

\section{Tiempo de juego}

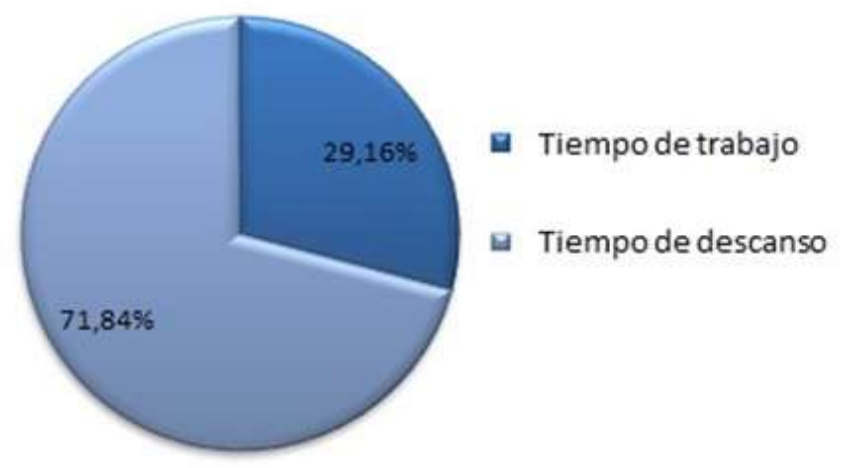

Figura 2. Distribución del \% tiempo de juego.

En la tabla 3, se presentan los valores descriptivos FC, \%FC máx, así como el RPE de los jugadores con cada tipo de pelota. La FC media y \% de $\mathrm{FC}_{\text {máx }}$ fueron significativamente más altas $(p<0,001)$ en los partidos jugados con pelota oficial (145 ppm, 72.5\% FC máx) en comparación el uso de pelotas de baja presión (140 
ppm, 69.9\% FC máx). No se encontraron diferencias estadísticamente significativas en la RPE en función del tipo de pelota utilizados.

Tabla 3. Valores mínimos (Mín), máximos (Máx), medios (M), y desviación típica (DT) en los datos extraídos de la $F C, \% F C_{\text {máx y }} R P E$ con cada tipo de pelota

\begin{tabular}{ccccccccccc}
\hline & \multicolumn{9}{c}{ Pelota oficial } & \multicolumn{7}{c}{ Pelota baja presión } \\
\cline { 2 - 11 } & Mín & Máx & M & DT & Mín & Máx & M & DT & Dif. & p \\
\cline { 2 - 11 } FC & 96,00 & 206,00 & 145,43 & 17,66 & 97,00 & 198,00 & 140,19 & 17,66 & 5,24 & $<0,001$ \\
\%FC máx & 48,10 & 102,49 & 72,47 & 8,75 & 48,43 & 98,51 & 69,85 & 8,75 & 2,62 & $<0,001$ \\
RPE & 11,00 & 20,00 & 13,81 & 2,46 & 10,00 & 19,00 & 13,75 & 2,18 & 0,06 & 0,940 \\
\hline
\end{tabular}

Leyenda: FC: Frecuencia cardiaca; \%FC máx: Porcentaje de la Frecuencia cardiaca máxima; RPE: Percepción subjetiva del esfuerzo.

En la figura 3 se observa el porcentaje de participación en cada zona de intensidad con la pelota normal y la pelota de baja presión. Como se puede observar, cerca del $30 \%$ del tiempo de juego los jugadores que disputaron los sets con pelotas normales estuvieron trabajando en zonas de baja y muy baja intensidad, mientras que aquellos que jugaron con pelota normal estuvieron únicamente un $20 \%$ del tiempo en esas zonas. Por el contrario, los partidos con pelota normal registraron un porcentaje del tiempo mayor en las zonas moderada, vigorosa y máxima, respecto a la pelota de baja presión. 


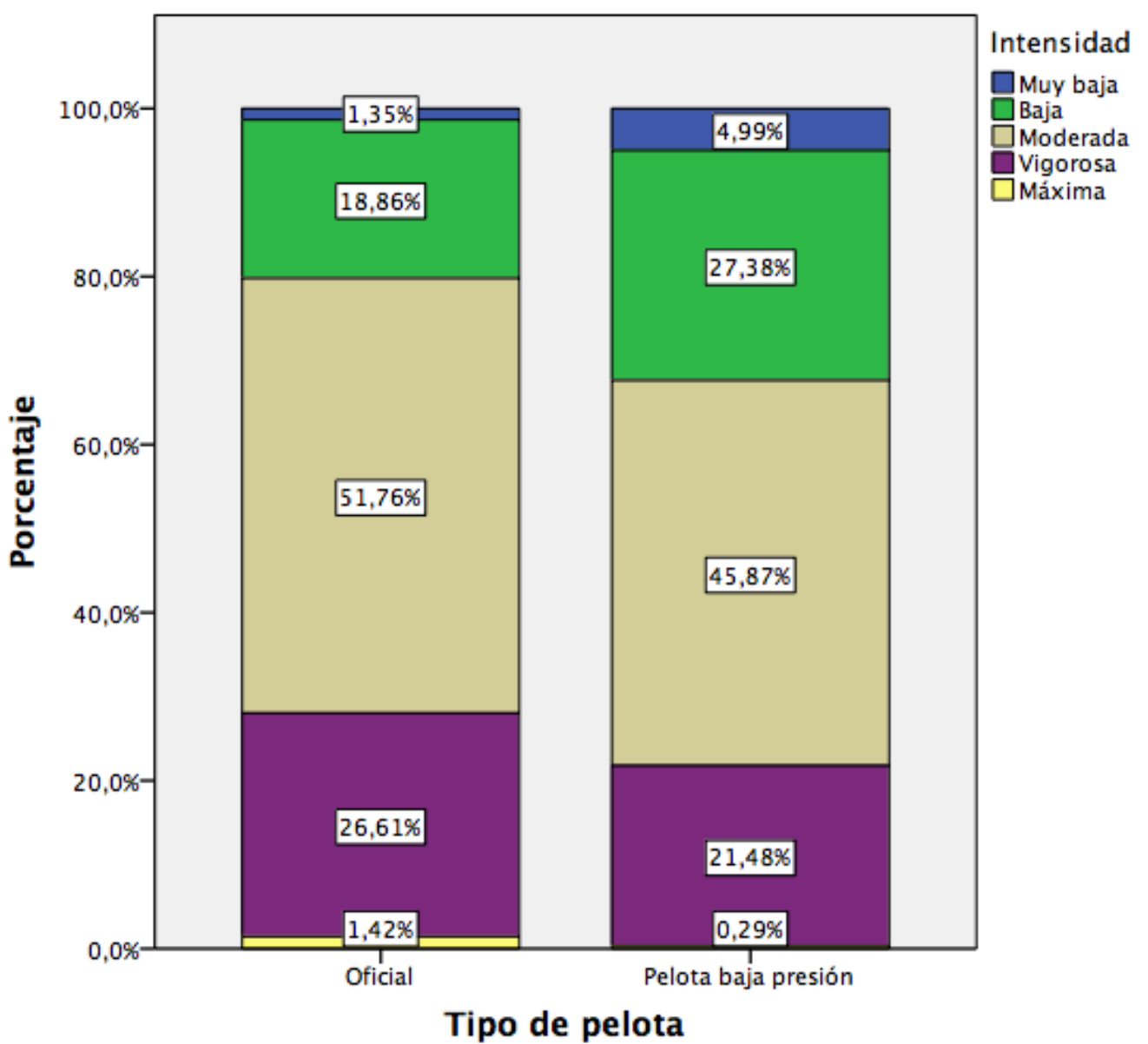

Figura 3. Distribución del \% de participación en cada zona de trabajo

En la tabla 4, se presentan los valores descriptivos medios (M) y desviación típica (DT) así como las diferencias por cada rango de actividad comparando el uso de la pelota normal y de baja presión. Como se puede observar, no se encontraron diferencias estadísticamente significativas en el tiempo expuesto por los jugadores en cada zona según el tipo de pelota. La mayor diferencia sobre el tiempo empleado se observa en la zona moderada (65-76\% FC máx), siendo de 12,82 minutos con la pelota normal, y de 10,94 minutos con la pelota de baja presión. 
Tabla 4. Diferencias en el tiempo de actividad (en minutos) en cada zona en según tipo de pelota.

\begin{tabular}{|c|c|c|c|c|c|c|c|c|}
\hline \multirow[b]{2}{*}{ \% FC máx } & \multicolumn{2}{|c|}{ Resumen } & \multicolumn{2}{|c|}{ Pelota normal } & \multicolumn{2}{|c|}{$\begin{array}{l}\text { Pelota baja } \\
\text { presión }\end{array}$} & \multirow[b]{2}{*}{ Dif } & \multirow[b]{2}{*}{$p$} \\
\hline & M & DT & $M$ & DT & $M$ & DT & & \\
\hline Muy baja ( $\leq 57 \%)$ & 0,76 & 1,64 & 0,33 & 0,59 & 1,19 & 2,20 & $-0,856$ & 0,143 \\
\hline Baja (58-64\%) & 5,60 & 5,61 & 4,67 & 4,78 & 6,53 & 6,35 & $-1,861$ & 0,357 \\
\hline Moderada (65-76\%) & 11,88 & 5,18 & 12,82 & 4,82 & 10,94 & 5,51 & 1,873 & 0,314 \\
\hline Vigorosa (77-95\%) & 5,86 & 7,65 & 6,59 & 8,15 & 5,12 & 7,31 & 1,465 & 0,596 \\
\hline Máxima ( $\geq 90 \%)$ & 0,21 & 1,00 & 0,35 & 1,41 & 0,07 & 0,28 & 0,282 & 0,438 \\
\hline
\end{tabular}

Rangos sobre el \% FC máx a partir del ACSM (2014).

En la figura 4, se presenta el porcentaje de respuesta de los jugadores, según la satisfacción percibida cuando jugaron con pelota normal o con pelota de baja presión. Como se puede observar, la satisfacción de los jugadores es mayor en los sets disputados con bola de baja presión que en los disputados con bola normal. De este modo, más del $60 \%$ de los jugadores mostraron una mejor percepción de diversión, comodidad y facilidad de juego y una mayor duración de los puntos en los sets disputados con pelotas de baja presión.

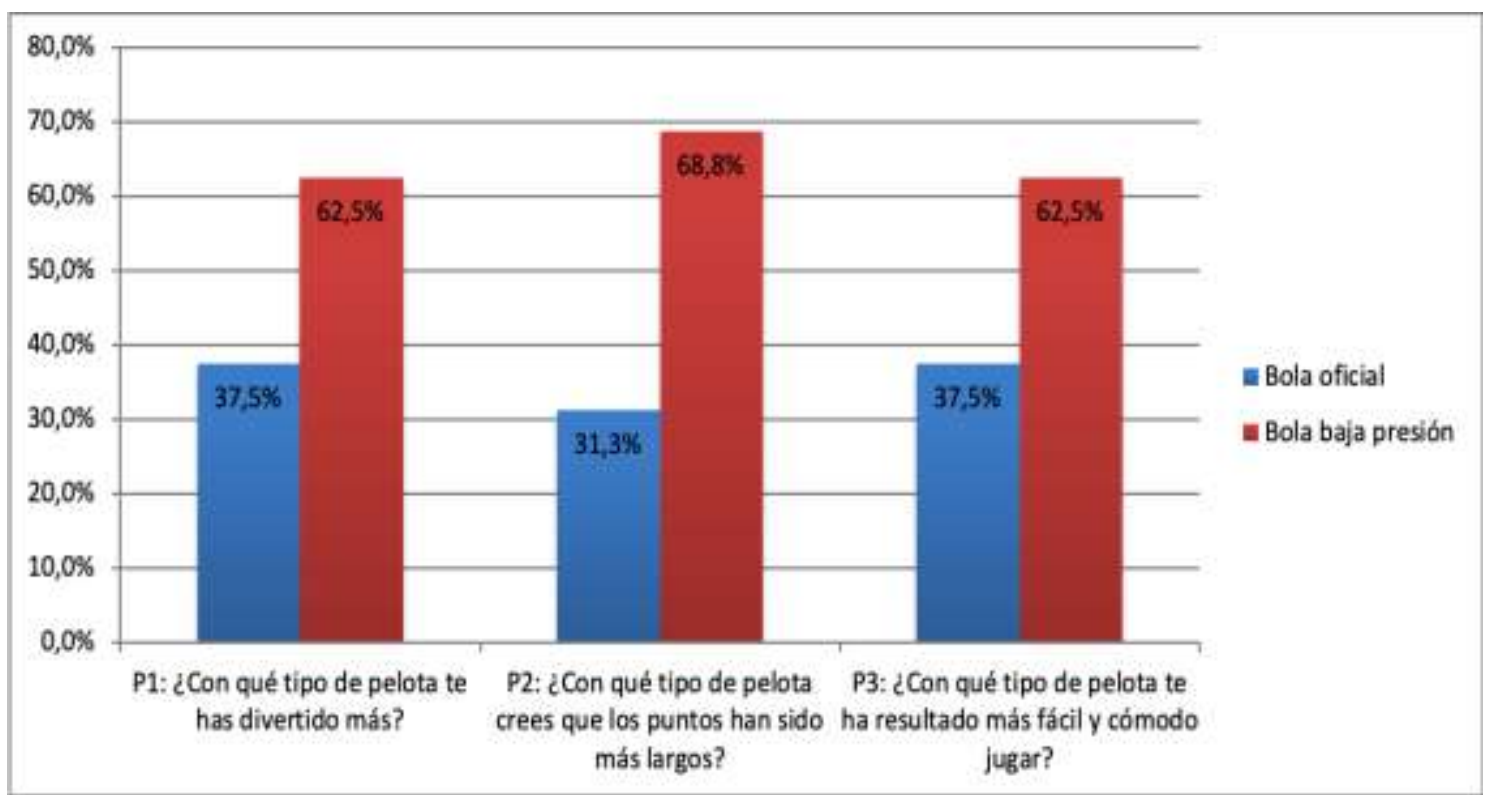

Figura 4. Satisfacción de los jugadores según el tipo de pelota.

\section{DISCUSIÓN}

Un mejor conocimiento de las características temporales y las demandas físicas de un partido de pádel en categorías de formación permitiría a los entrenadores y jugadores a tomar decisiones acordes a parámetros representativos de la competición. El objetivo de este estudio fue conocer las alteraciones en la 
estructura temporal y las demandas físicas en un partido de pádel en función del uso de pelotas oficiales y de pelotas de baja presión.

\section{Estructura temporal.}

Los datos de este estudio muestran una duración del set de 24,03 minutos en los sets analizados con pelota oficial, y 23,38 minutos con pelota de baja presión (tabla 1). Estos resultados son inferiores a los 32 minutos de tiempo total de un set en jugadores profesionales (Almonacid, 2012) o los 30 minutos de partidos oficiales de nivel regional de jugadores adolescentes (Sánchez-Alcaraz, 2014a).

La duración media de los puntos, tanto con pelota oficial como con baja presión, fue de 8 segundos (tabla 2). Estos valores son inferiores a los obtenidos en los partidos del circuito Pádel Pro Tour en jugadores profesionales (Althaus, 2012) o partidos oficiales de nivel regional (Sánchez-Alcaraz, 2014a), pero similares a los resultados mostrados por jugadores adolescentes de categoría nacional (Sañudo et al., 2008). Parece por tanto que la duración del punto se ve afectada por el nivel y edad. Sin embargo, y en contra de lo observado en tenis (Kachel et al., 2014), el uso de una pelota de baja presión no afectó a la duración del punto en pádel en etapas de formación.

El número medio de golpes por punto fue de 4,3, tanto con pelota oficial como con pelota de baja presión (tabla 2). Estos valores son inferiores a los 6,7 golpes por punto obtenidos en jugadores jóvenes de nivel regional (Sánchez-Alcaraz, 2014a). Asimismo, el número de golpes aumenta en jugadores masculinos y femeninos profesionales, con una media de 9,4 y 9,1 golpes por punto respectivamente (Sánchez-Alcaraz, 2014b). En este sentido, los jugadores profesionales poseen un mayor dominio de la técnica y una mejor condición física, lo que permite realizar un mayor número de intercambio de golpes y jugar peloteos más largos que los jugadores no profesionales o en etapas formación (Sánchez-Alcaraz, 2013b; Sánchez-Alcaraz, Orozco, Courel-Ibañez y SánchezPay, 2018). En relación al número de golpes con y sin bote, no se han encontrado diferencias significativas entre el uso de pelota oficial o de baja presión (tabla 2). Es interesante destacar que los jugadores jóvenes de pádel realizaron golpes sin bote en el $16 \%$ de las ocasiones, siendo valores muy inferiores a la distribución en pádel profesional (Courel-lbáñez, Sánchez-Alcaraz, y Muñoz, 2017). Estas diferencias sugieren que los jugadores de nivel de iniciación emplean la mayor parte del tiempo en la zona de fondo de la pista, alejados de la red. Sin embargo, no podemos proporcionar datos para confirmar esta hipótesis. En cuanto al porcentaje del tiempo de juego, se puede observar un tiempo de trabajo inferior al 30\% respecto al tiempo total de juego en jóvenes jugadores de pádel (figura 2). Los resultados obtenidos son inferiores a los de Almonacid (2012) y Pradas et al. (2012) en pádel senior masculino y femenino, pero superiores al tenis, que varía entre el $20 \%$ y $26 \%$ del tiempo total de juego (Christmass, Richmond, Cable y Hartmann, 1995; Christmass, Richmond, Cable, Arthur, y Harmann, 1998; Ferrauti, Weber y Wright, 2003; Kovacs, 2004; Morante y Brotherhood, 2005; Smekal, Von Duvillard, Rihacek, et al., 2001). En consistencia con los resultados anteriores, estos valores inferiores pueden 
deberse al nivel de juego de los participantes, limitando su capacidad de mantener un intercambio de golpes continuo superior a los 5 golpeos.

\section{Niveles de actividad física}

La FC media durante el partido de pádel aumentó cuando se utilizó pelota oficial (145 ppm, 72,5\% FC máx) y disminuyó con el uso de pelota de baja presión (140 ppm, 69,9\% FC máx) en jugadores jóvenes de iniciación (tabla 3). Estos resultados son ligeramente inferiores a los encontrados un estudio previo sobre demandas de la competición en jugadores jóvenes de pádel en competición, donde la FC media fue de 148 ppm, un 74,1\% respecto a la FC máxima (Sañudo et al., 2008). La percepción subjetiva del esfuerzo (RPE) mostró unos valores medios de 13,8 en los sets disputados tanto con pelota oficial como de baja presión (tabla 3). Estos valores pueden interpretarse como un esfuerzo "algo duro" en la escala de Borg sobre 20 puntos. Estos resultados son muy similares a los encontrados en partidos de pádel (Amieba y Salinero, 2013) y tenis (Fernández-Fernández, Méndez-Villanueva, Fernández-García, y Terrados, 2007). El porcentaje de participación en cada rango de intensidad (figura 3) así como el tiempo en minutos que emplean los jugadores en las diferentes zonas (tabla 4) no mostró diferencias según el tipo de pelota usada. No obstante, se observa que el uso de una pelota oficial incrementó el tiempo de participación en las zonas de moderada y vigorosa intensidad hasta el $78 \%$, mientras que el uso de una pelota de baja presión solo alcanzó un $67 \%$. Estas diferencias van en relación a las ya observadas sugiriendo que el uso de pelotas de baja presión parece disminuir la intensidad del partido de pádel. Sin embargo, los niveles de actividad física mostrados por los niños durante un partido de pádel pueden ser considerados como suficiente según las recomendaciones del ACSM (2014), pudiéndose recomendar la práctica del pádel como una actividad física saludable (MuñozMarín et al., 2019).

Los jugadores jóvenes de pádel mostraron una mayor predisposición a jugar con pelota de baja presión que con pelota oficial (figura 4). El uso de una pelota de baja presión proporcionó a los niños una mayor diversión y les resultó más cómodo jugar. Estos datos pueden favorecer a disminuir las sobrecargas y esfuerzos y así disminuir lesiones y dolencias que puedan acarrear el abandono de la práctica del pádel (Sánchez-Alcaraz, Courel-lbáñez, Díaz-García y MuñozMarín, 2019). Además, percibieron que los puntos durante el partido habían sido más largos con el uso de pelotas de baja presión. Aunque existen estudios que indican que la percepción de la satisfacción del jugador de pádel es mayor cuando aumenta el número de golpeos en la tarea (Sánchez-Alcaraz, SánchezPay, Gómez-Mármol, Bazaco- Belmonte, y Molina-Saorín, 2017) en nuestro caso, la mayor satisfacción de los jugadores puede deberse a aspectos relacionados con la sensación de golpeo, control de direcciones de la pelota, 0 la simple novedad en el uso de una pelota diferente.

Este estudio posee una serie de limitaciones que son necesarias exponer. Por un lado, la juventud e inexperiencia de los jugadores ha podido influir en la fiabilidad de los datos obtenidos. Además, los jugadores estaban acostumbrados 
a utilizar pelotas oficiales, por lo que hubiera sido interesante incluir un análisis del partido tras la práctica continuada previamente con cada tipo de pelota. Para futuras líneas de investigación sería apropiado aumentar el número de niños objeto de estudio. Además, sería interesante confirmar si el uso de otras modificaciones como jugar en una pista de tamaño reducido produce los mismos efectos sobre la intensidad, dinámicas de juego y diversión en jugadores de pádel en etapas de formación.

\section{CONCLUSIONES}

Con los resultados obtenidos en este trabajo, se pueden establecer las siguientes conclusiones:

- La duración media del set en pádel de formación es aproximadamente de 24 minutos, con una media aproximada de 50 puntos y más de 200 golpes por set.

- Los jugadores realizan 4 golpes de media por punto, con mayor cantidad de golpes con bote que sin bote, y con una duración media de cada punto de 8 segundos aproximadamente.

- El tiempo de trabajo representa el $30 \%$ del tiempo total del partido.

- Los jugadores tienen una FC media de 140-145 ppm lo que representa un $67-69 \%$ de la $\mathrm{FC}_{\text {máx. }}$ Los valores de RPE medios son cercanos a 13 puntos en la Escala de Borg.

- El tipo de pelota no parece afectar a la estructura temporal de un partido de pádel. Sin embargo, el uso de una pelota de baja presión parece disminuir la intensidad del juego.

- Los niveles de actividad física de un partido de pádel en la iniciación se encuentran dentro de los rangos para ser considerado una actividad saludable a nivel cardiovascular.

- Los jóvenes jugadores que se inician a la práctica del pádel tienen una mayor percepción de diversión, facilidad y comodidad en el juego y una mayor duración de los puntos cuando juegan con pelotas de baja presión.

\section{REFERENCIAS BIBLIOGRÁFICAS}

American College of Sports Medicine. (2014). ACSM's guidelines for exercise testing and prescription. Lippincott Williams \& Wilkins.

Almonacid, B. (2012). Perfil de juego en pádel de alto nivel. Tesis Doctoral. Universidad de Jaén.

Althaus, A. (2012). Pádel. Estadísticas y preparación física. Valladolid: Artes Gráficas Gar Cue.

Amieba, C., y Salinero, J. J. (2013). Overview of paddle competition and its physiological demands. AGON. International Journal of Sport Sciences, 3(2), 60-67.

Borg, E., y Kaijser, L. (2006). A comparison between three rating scales for perceived exertion and two different work tests. Scandinavian Journal of Medicine \& Science in Sports, 16(1), 57-69. https://doi.org/10.1111/j.1600$\underline{0838.2005 .00448 . x}$ 
Buszard, T., Reid, M., Masters, R., y Farrow, D. (2016). Scaling the equipment and play area in children's sport to improve motor skill acquisition: A systematic review. Sports Medicine, 46(6), 829-843. https://doi.org/10.1007/s40279-015$\underline{0452-2}$

Cabello, D., y Torres, G. (2004). Características de la competición en tenis y bádminton. Investigación en deportes de raqueta: tenis y bádminton. Murcia: Universidad Católica de San Antonio.

Christmass, J.L., Richmond, S.E., Cable, N., y Hartmann. P.E. (1995). A metabolic characterisation of single tennis. In: T. Reilly, M. Hughes, A. Lees. Science and Racket Sports I, (pp. 3-9). London, England: E \& Fn Spon.

Christmass, M., Richmond, S., Cable, N., Arthur, P., y Hartmann, P. (1998). Exercise intensity and metabolic response in singles tennis. Journal of Sports Sciences, 16, 739-747. https://doi.org/10.1080/026404198366371

Courel-Ibáñez, J. y Sánchez-Alcaraz, B.J. (2017). Efecto de las variables situacionales sobre la duración y eficacia de los puntos en jugadores de pádel de élite. Apunts, Educación Física y Deportes, 127, 72-78. https://doi.org/10.5672/apunts.20140983.es.(2017/1).127.07

Courel-Ibáñez, J., y Sánchez-Alcaraz, B.J. (2018). The role of hand dominance in padel: performance profiles of professional players. Motricidade, 14(4), 33-41. https://doi.org/10.6063/motricidade.14306

Courel-Ibáñez, J., Sánchez-Alcaraz, B.J., y Cañas, J. (2017). Game performance and length of rally in professional padel players. Journal of Human Kinetics, 55, 161169. https://doi.org/10.1515/hukin-2016-0045

Courel-lbáñez, J., Sanchez-Alcaraz, B.J., Muñoz-Marín, D. (2017). Exploring game dynamics in padel. Implications for assessment and training. The Journal of Strength and Condition Ressearch. https://doi.org/10.1519/JSC.0000000000002126

Courel-lbáñez, J., Sánchez-Alcaraz, B.J., García, S., y Echegaray, M. (2017). Evolución del pádel en España en función del género y edad de los practicantes. Cultura, Ciencia y Deporte, 34(12) 39-46. https://doi.org/10.12800/ccd.v12i34.830

Diaz, V. J. L., Cantó, E. G., y Soto, J. J. P. (2013). Nivel de ejecución del remate de potencia de pádel en alumnos de la Universidad de Murcia. EmásF: Revista Digital de Educación Física, (23), 16-24

Farrow, D., y Reid, M. (2010). The effect of equipment scaling on the skill acquisition of beginning tennis players. Journal of Sports Sciences, 28(7), 723-732. https://doi.org/10.1080/02640411003770238

Fernández-Fernández, J., Méndez-Villanueva, A., Fernández-Garcia, B., y Terrados, N. (2007). Match activity and physiological responses during junior female singles tennis tournament. British Journal of Sport Medicine, 41(11), 711-716. https://doi.org/10.1136/bjsm.2007.036210

Ferrauti, A., Weber, K., y Wright, P.R. (2003) Endurance: basic, semi-specific and specific. In: M. Reid, A. Quinn, M.Crespo. Strength and conditioning for tennis, (pp. 93-111). London, England: ITF.

Gabin, B., Camerino, O., Anguera, M.T., y Castañer, M. (2012). Lince: Multiplatform sport analysis software. Procedia - Social and Behavioral Sciences, 46, 46924694. https://doi.org/10.1016/j.sbspro.2012.06.320

Kachel, K., Buszard, T., y Reid, M. (2015). The effect of ball compression on the matchplay characteristics of elite junior tennis players. Journal of Sports Sciences, 33(3), 320-326. https://doi.org/10.1080/02640414.2014.942683

Kovacs, M. (2004). A comparison of work/rest intervals in men's professional tennis. Medicine and Science in Tennis, 9(3): 10-11. 
Lacasa, K., Orteub, E., Gabriel, E., Torrents, C., y Salas, C. (2017). Las situaciones reducidas de juego como constreñimiento de la tarea en la iniciación al pádel para niños de seis a diez años. Revista Andaluza de Medicina del Deporte, 10(3), 158-162. https://doi.org/10.1016/..ramd.2016.06.010

Landis, J. R., y Koch, G. G. (1977). The measurement of observer agreement for categorical data. Biometrics, 159-174.

Larson, E. J., y Guggenheimer, J. D. (2013). The effects of scaling tennis equipment on the forehand groundstroke performance of children. Journal of Sports Science \& Medicine, 12(2), 323-331.

Limpens, V., Buszard, T., Shoemaker, E., Savelsbergh, G. J., y Reid, M. (2018). Scaling constraints in junior tennis: the influence of net height on skilled players' matchplay performance. Research Quarterly for Exercise and Sport, 89(1), 1-10. https://doi.org/10.1080/02701367.2017.1413230

Machado, F. A., \& Denadai, B. S. (2011). Validity of maximum heart rate prediction equations for children and adolescents. Arquivos Brasileiros de Cardiologia, 97(2), 136-140. https://doi.org/10.1590/S0066782X2011005000078

Morante, S., y Brotherhood, J. (2005). Match Characteristics of Professional Singles Tennis. Medicine and Science in Tennis, 10(3), 12-13.

Muñoz, D., Díaz, J., Pérez-Quintero, M., Grijota, F.J., Courel-lbáñez, J., y SánchezAlcaraz, B.J. (2019). Efectos del volumen de práctica de pádel sobre la composición corporal en jugadores amateurs. Acción Motriz, 22, 17-22.

Muñoz-Marín, D., Sánchez-Alcaraz, B.J., Courel-lbáñez, J., Romero, E., Grijota, F.J., y Díaz, J. (2016). Estudio sobre el perfil y distribución de las pistas de pádel en la Comunidad Autónoma de Extremadura. E-Balonmano.com: Revista de Ciencias del Deporte, 12(3), 223-230.

Pradas, F., Castellar, C., Coll, I. y Rapún, M. (2012). Análisis de la estructura temporal del pádel femenino. IV Congreso Internacional de Ciencias del Deporte y la Educación Física. Pontevedra, España.

Sánchez-Alcaraz, B.J. (2013a). Historia del pádel. Materiales para la historia del deporte, 11, 57-60.

Sánchez-Alcaraz, B.J. (2013b). Táctica del pádel en la etapa de iniciación. Trances: Revista de Transmisión del Conocimiento Educativo y de la Salud, 5(1), 109-116.

Sánchez-Alcaraz, B.J. (2013c). Analysis of the temporary aspects and actions of the game in performance junior players and the differences when playing with adapted material. Coaching and Sport Science Review, 61 (21), 29-30.

Sánchez-Alcaraz, B. J. (2014a). Análisis de la exigencia competitiva del pádel en jóvenes jugadores. Kronos, 13(1), 1-7.

Sánchez-Alcaraz, B. J. (2014b). Diferencias en las acciones de juego y la estructura temporal entre el pádel masculino y femenino profesional. Acción Motriz, 12, 1735.

Sánchez-Alcaraz, B.J., Cañas, J., y Courel, J. (2015). Análisis de la investigación científica en pádel. AGON. International Journal of Sport Sciences, 5 (1), 44-54.

Sánchez-Alcaraz, B.J., Courel-lbáñez, J., y Cañas, J. (2018). Estructura temporal, movimientos en pista y acciones de juego en pádel: Revisión sistemática. Retos, Nuevas Tendencias en Educación Física y Deportes, 33, 129-133. https://doi.org/10.47197/retos.v0i33.55025

Sánchez-Alcaraz, B.J., Courel-Ibáñez, J., Díaz, J., Grijota, F.J., y Muñoz, D. (2019). Efectos de la diferencia en el marcador e importancia del punto sobre la estructura temporal en pádel. Journal of Sport and Health Research, 11(2), 139150. 
Sánchez-Alcaraz, B.J., Courel-lbáñez, J., Díaz-García, J., y Muñoz-Marín, J. (2019). Estudio descriptivo de lesiones de pádel: relación con el género, edad, nivel de los jugadores y localización de las lesiones. Revista Andaluza de Medicina del Deporte, 12(1), 29-34. https://doi.org/10.33155/j.ramd.2018.02.004

Sánchez-Alcaraz, B.J., Orozco, V., Courel-Ibáñez, J., Sánchez-Pay, J. (2018). Evaluación de la velocidad, agilidad y fuerza en jóvenes jugadores de pádel. Retos, Nuevas Tendencias en Educación Física y Deportes, 34, 263-266. https://doi.org/10.47197/retos.v0i34.60463

Sánchez-Alcaraz, B.J., Sánchez-Pay, A., Gómez-Mármol, A., Bazaco-Belmonte, M.J., y Molina- Saorín, J. (2017). Diferencias en la forma de organización de las sesiones de pádel con estudiantes. Revista Internacional de Medicina y Ciencias de la Actividad Física y el Deporte, 17(67), 467-479. https://doi.org/10.15366/rimcafd2017.67.005

Sánchez-Pay, A., Sánchez-Alcaraz., y Courel-lbáñez, J. (2018). Propuesta metodológica de adaptación al entrenamiento del tenis en la etapa de iniciación. TRANCES: Revista de Transmisión del Conocimiento Educativo y de la Salud, 10(3), 253-266.

Sañudo, B., De Hoyo, M., y Carrasco, L. (2008). Demandas fisiológicas y características estructurales de la competición en pádel masculino. Apunts. Educación Física y Deportes, 94, 23-28.

Smekal, G., Von Duvillard, S., Rihacek, C., Pokan, R., Hofmann, P., Baron, R., Tschan, H., y Bachl, N. (2001). A physiological profile oftennis match play. Medicine Science in Sports and Exercise, 33(6): 999 - 1005. https://doi.org/10.1097/00005768-200106000-00020

Tanaka, H., Monahan, K. D., y Seals, D. R. (2001). Age-predicted maximal heart rate revisited. Journal of the American College of Cardiology, 37(1), 153-156. https://doi.org/10.1016/S0735-1097(00)01054-8

Timmerman, E., De Water, J., Kachel, K., Reid, M., Farrow, D., y Savelsbergh, G. (2015). The effect of equipment scaling on children's sport performance: the case for tennis. Journal of Sports Sciences, 33(10), 1093-1100. https://doi.org/10.1080/02640414.2014.986498

Torres-Luque, G, Ramirez, A, Cabello-Manrique, D, Nikolaidis, P.T., y Alvero-Cruz, J.R. (2015). Match analysis of elite players during paddle tennis competition. International Journal of Performance Analysis in Sport, 15(3),1135-1144. https://doi.org/10.1080/24748668.2015.11868857

\section{Referencias totales / Total references: 46 (100\%) \\ Referencias propias de la revista / Journal's own references: 1 (2,17\%)}

Rev.int.med.cienc.act.fís.deporte - vol. 20 - número 79 - ISSN: 1577-0354 